Advance Journal of Food Science and Technology 5(6): 787-792, 2013

DOI:10.19026/ajfst.5.3164

ISSN: 2042-4868; e-ISSN: 2042-4876

(C) 2013 Maxwell Scientific Publication Corp.

Submitted: March 01, $2013 \quad$ Accepted: March 27, 2013

Published: June 05, 2013

\title{
Research Article \\ Effects of Shading Stress and Light Recovery on the Photosynthesis Characteristic and Chlorophyll Fluorescence Characteristic of Fragaria Ananassa Duch. cv. Toyonoka
}

\author{
${ }^{1,2}$ Renyan Duan, ${ }^{1}$ Minyi Huang, ${ }^{1}$ Zhigao Wang, ${ }^{1}$ Zhongxin Zhang and ${ }^{2}$ Weiyi Fan \\ ${ }^{1}$ School of Life Science, Anqing Teachers College, Anqing, Anhui 246011, China \\ ${ }^{2}$ College of Life Science, Shaanxi Normal University, Xi'an, 710062, China
}

\begin{abstract}
Light is an important resource for plant growth and development, crops need to change their physiological characteristics to different light environments. Fragaria ananassa Duch. cv. Toyonoka. is an important economic plant which is widely planted at home. A greenhouse experiment was conducted from April 2010 with different sun-shading treatment, $85 \%(\mathrm{CK}), 60 \%\left(\mathrm{~T}_{1}\right), 35 \%\left(\mathrm{~T}_{2}\right)$ and $10 \%\left(\mathrm{~T}_{3}\right)$. After 7 days of shading stress, the physiological characteristics were slowly recovered. The results showed that (1) Under shading condition, Light saturation rate (Amax), Apparent Quantum Yield (AQY), Carboxylation Efficiency (CE), dark respiration (Rd), Light Saturation Point (LSP) and Light Compensation Point (LCP) became lower. (2) The maximal fluorescence (Fm), light energy transformation efficiency of PS II (Fv/Fm), actual photochemical efficiency of PS II in the light (Yield), photochemical quenching coefficient (qP) declined with shading stress increase. (3) There were significant difference in the chlorophyll fluorescence parameters among different treatment groups by the end of sun-shading treatment $(\mathrm{p}<0.05)$. After the light was recovered, the physiological characteristics could rapidly recover under low shading stress and moderate shading stress, while in severe shading stress the physiological characteristics hardly recover.
\end{abstract}

Keywords: Chlorophyll fluorescence, Fragaria ananassa, light recovery, shanding stress

\section{INTRODUCTION}

There is a broad consensus in ecology that light is an important resource for the survival, growth and distribution of crops (Jensen et al., 2012). In different time and space conditions, the Photosynthetic Photon Flux Density (PPFD) is different, and crops need to develop acclimation and plasticity mechanism to cope with the varying light regimes by changing their morphological and physiological adaptations to different light environments (Gianoli and Valladares, 2012; Wyka et al., 2012).

During planting, most crops are exposed to different light gradients (from full shade to full exposure to sun light), and plants are easily subjected to at least some degree of shade. In response to shading, examples for an active type of plasticity are an increase in specific leaf area (Delagrange, 2011), in leaf size (Valladares and Pearcy, 1998), or the elongation of internodes (Van Kleunen and Fischer, 2005). In order to help balance the light absorption between the two photo-systems (PSI and PSII), shade leaves have a lower chlorophyll a/b ratio (Chazdon, 1992). In lower light environment, crops may change leaf structure and physiology, which determines the leaf carbon acquisition and gas exchange (Gianoli and Valladares, 2012; Wyka et al., 2012).
Some studies on response of photosynthetic plasticity to changed light environments mainly focused on specific light environment, such as artificial deadlines different dense shade at laboratory experiment (Feijó et al., 2009) and some patches (such as gap and non-gap; gap, non-gap and expanding gap) at outdoor experiment (Shimatani and Kubota, 2011). However, previous studies on response of photosynthetic plasticity to different stable light environments have failed to dynamic changes of light environment, such as shading stress and light recovery (Oguchi et al., 2006; Feijó et al., 2009; Shimatani and Kubota, 2011). So, further studies are still essential.

Chlorophyll fluorescence technique provides a rapid and non-intrusive probe for the measurement of photosynthetic function under different light condition in recent years (Razavi et al., 2008). Fragaria ananassa Duch. cv. Toyonoka is an important economic plant which is widely planted at home and abroad, and easily suffers the effect of light stress. It was widely cultivated in our country. How does its physiological process react with the changing light environment? How and in what extent does its physiological plasticity response to change of light environment and light recovery? Based on our observations, we hypothesize that:

Corresponding Author: Renyan Duan, School of Life Science, Anqing Teachers College, Anqing, Anhui 246011, China, Tel.: 13866092294

This work is licensed under a Creative Commons Attribution 4.0 International License (URL: http://creativecommons.org/licenses/by/4.0/). 
- The physiological process of $F$. ananassa is sensitive to changes in the light environment.

- It is be easily recovered in lower shading stress after the light was recovered, while it is hardly recovered in severer shading stress. The objective of our study is to elucidate the relationship between photosynthetic plasticity and light environment of the $F$. ananass $a$ and to test the above hypotheses.

\section{MATERIALS AND METHODS}

The experiment was conducted at Anqing Botanical Garden, which is located in the western suburb of Anqing. These seedlings of one year-old originally were grown in a greenhouse in the early spring of 2010. Then they were transferred to a control light (about $85 \%$ of full ambient light) at the beginning of April 2010. At the end of April of 2008, these potted seedlings were divided at random into four groups, with each group comprising three potted seedlings. Each of the three groups was subjected to one of the four different light environments (control-, mild-, moderateand severe-stress). The seedlings were irrigated at regular periods depending on the weather and soil moisture status.

Methods: For assessment of light effects on the physiological characteristics of $F$. ananassa, a light gradient was established using different layers of neutral gauze to provide four levels of visible light: control light (CK, about $85 \%$ of full ambient sunlight), mild stress $\left(\mathrm{T}_{1}\right.$, about $60 \%$ of full ambient sunlight), moderate stress $\left(\mathrm{T}_{2}\right.$, about $35 \%$ of full ambient sunlight), severe stress $\left(\mathrm{T}_{3}\right.$, about $10 \%$ of full ambient sunlight). After 7 days of light stress, the lights of seedlings were recovered until the 11 th day.

Measurements of net photosynthetic rate $\left(P_{n}\right)$, stomata conductance $\left(g_{s}\right)$, transpiration rate $\left(T_{r}\right)$, intercellular $\mathrm{CO}_{2}$ concentration $\left(C_{i}\right)$, respiratory rate etc. were carried out under ambient/controlled conditions with a portable photosynthesis measurement system (LI-6400, LI-COR Inc. Lincoln, NE, USA). A $2 \times 3 \mathrm{~cm}$ chamber was used and a LED 6400-02B lamp provided the light source.

All measurements were performed from 8:00 to 11:00 am. Light-response curves were created with eleven levels of PPFD $(0,15,25,50,100,200,400$, $600,800,1,000,1,200 \mu \mathrm{moL}$ photons $\left./ \mathrm{m}^{2} . \mathrm{s}\right)$ in decreasing order. The time allowed between each reading was 2-3 min intervals. All measured leaves were located in middle to upper parts of one fully expanded and mature leaf. Measurements were made at a temperature of $25^{\circ} \mathrm{C}$ and a $\mathrm{CO}_{2}$ concentration of 400 ppm. The Light saturation rate (Amax), Apparent Quantum Yield (AQY), Carboxylation Efficiency (CE), dark respiration $\left(R_{d}\right)$ and Light Compensation Point (LCP) was calculated (Wang et al., 2004). The sampled leaf was placed in the curette until at least five stable readings were obtained.

At various time intervals during the shading treatment and the recovery, the in vivo chlorophyll fluorescence of PSII was measured by a pulsemodulated fluorometer (PAM-2100, Walz, Effeltrich, Germany) connected to a computer with control software. For consecutive measurement of chlorophyll fluorescence parameters, we marked a fixed position in each sampled leaf. In order to measure the minimal Fluorescence $\left(F_{0}\right)$ and maximal fluorescence parameters $\left(F_{m}\right)$, the sample was first dark adapted for at least 20 min using a leaf clip. At nature light, we measure the minimal fluorescence $\left(F_{0}{ }^{\prime}\right)$ and maximal fluorescence parameters $\left(F_{m}{ }^{\prime}\right)$. The following fluorescence parameters were calculated: variable fluorescence (dark) $\left(F_{v}=F_{m}-F_{0}\right)$, maximal photochemical efficiency of PSII (dark adapted leaf) $\left(F_{v} \sqrt{ } F_{m}\right)$; variable fluorescence (light) $\left(F_{v}{ }^{\prime}=F_{m}{ }^{\prime}\right.$ $F_{0}{ }^{\prime}$ ), photochemical efficiency of PSII in the light, which is the efficiency if all reaction centers remain open $\left(F_{v}{ }^{\prime} / F_{m}{ }^{\prime}\right)$; actual photochemical efficiency of PSII in the light, which is the efficiency under the actual degree of reaction center closure $\left(\left(F_{m}{ }^{\prime}-/ F\right) / F_{m}{ }^{\prime}\right)$; the apparent Electron Transport Rate (ETR = $\left.F / F_{m} \times \mathrm{PPFD} \times 0.5 \times 0.84\right)($ Razavi et al., 2008).

There are two kinds of fluorescence quenching, such as photochemical quenching $(q P)\left(q P=/\left(F_{m}{ }^{\prime}-\right.\right.$ $\left.F) /\left(F_{m}{ }^{\prime}-F_{0}\right)\right)$ and non-photochemical quenching $(q N)$ $\left(q N=1-\left(F_{m}^{\prime}-F_{0}\right) /\left(F_{m}-F_{0}\right)=1-F_{v}^{\prime} / F_{v}\right)$.

All data were plotted using MICROSOFT EXCEL 2003, and each value of mean and S.D. (standard deviation) in the figures represents at least 3 replications of measurements. A one-way ANOVA was used to evaluate differences of chlorophyll fluorescence characteristics of F. Anamosa of shading stress and light recovery. Significant differences discussed have a probability $(p)$ value $<0.05$.

\section{RESULTS AND DISCUSSION}

In the weak intensity range $\left(0 \sim 600 \mu \mathrm{mol} / \mathrm{m}^{2} \cdot \mathrm{s}\right)$, the photosynthetic rate $\left(P_{n}\right)$ of both the shade and unshading leaves has a rapid increase. There were little differences between different treatments $(p>0.05)$. When the light intensity is greater than $600 \mu \mathrm{mol} / \mathrm{m}^{2} \cdot \mathrm{s}$, with the increase of light intensity, the photosynthetic rate $\left(P_{n}\right)$ tend to be stable, and the difference between the treatment increase. The photosynthetic rate of control leaves is higher significantly than that of the shade leaves $(\mathrm{p}<0.05)$ (Fig. 1).

This suggests that in low light intensity, shading has no significant influence on strawberry 


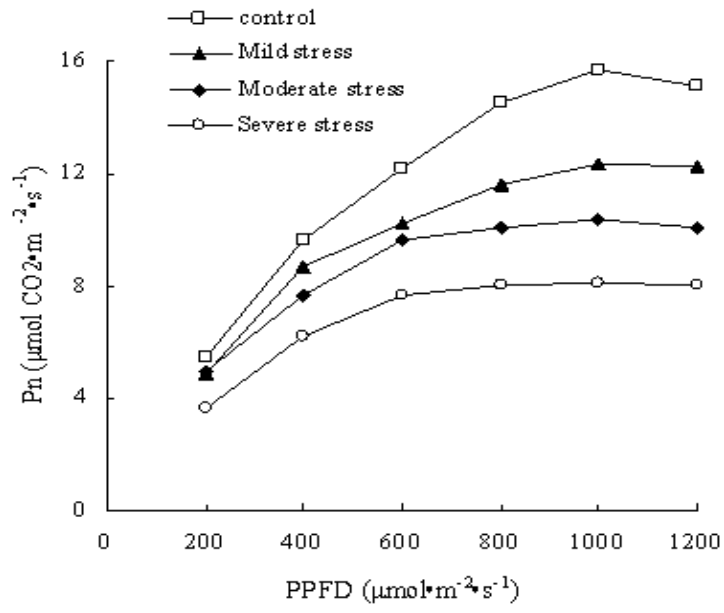

Fig. 1: Light-response curves under different shading stress

photosynthetic rate. However, with light intensity increase, the influence of the shade increases significantly.
In Fig. 2, compared with the nature light, different shading strength reduced significantly the Lightsaturated photosynthetic rate $\left(A_{\max }\right)$, Apparent Quantum Yield (AQY), Carboxylation Efficiency (CE), Light Saturation Point (LSP), Light Compensation Point (LCP) and dark respiration $\operatorname{rate}(R d)(\mathrm{p}<0.05)$, except some parameters, such as AQY and LCP had no significant difference under mild stress and moderate stress $(p>0.05)$. After the light was recovered, the physiological characteristics could rapidly recover under low shading stress and moderate shading stress, while in severe shading stress the physiological characteristics hardly recover (Fig. 2).

The $F_{0}$ is a fluorescence yield when PSII reaction center is totally open (Feijó et al., 2009). From picture 3-A, we know $F_{0}$ stays the same level basically when given normal light. Under the same shading stress, with the stress time increased, the change of $F_{0}$ which under moderate stress and severe stress has a certain turning, that is: it firstly expressed a slight decrease and then has a growing tendency. With the degree of stress serious, the turning point has an advance tendency.
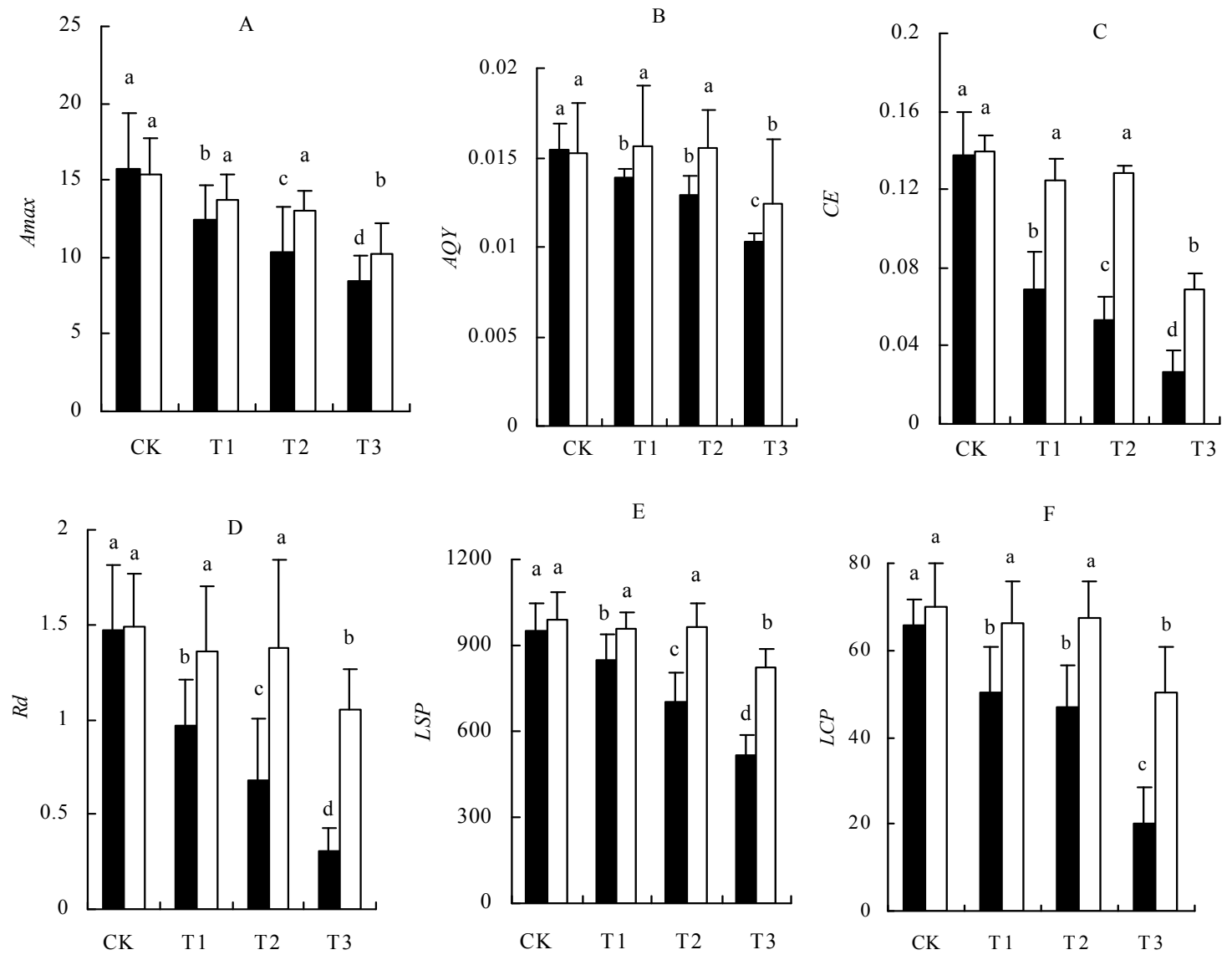

Fig. 2: Effects of shading stress and light recovery on the photosynthesis characteristic of Fragaria ananassa the different letters indicate statistically significant differences at $\mathrm{p}<0.05$ between elevations

Shading stress $\square$ Light recovery 

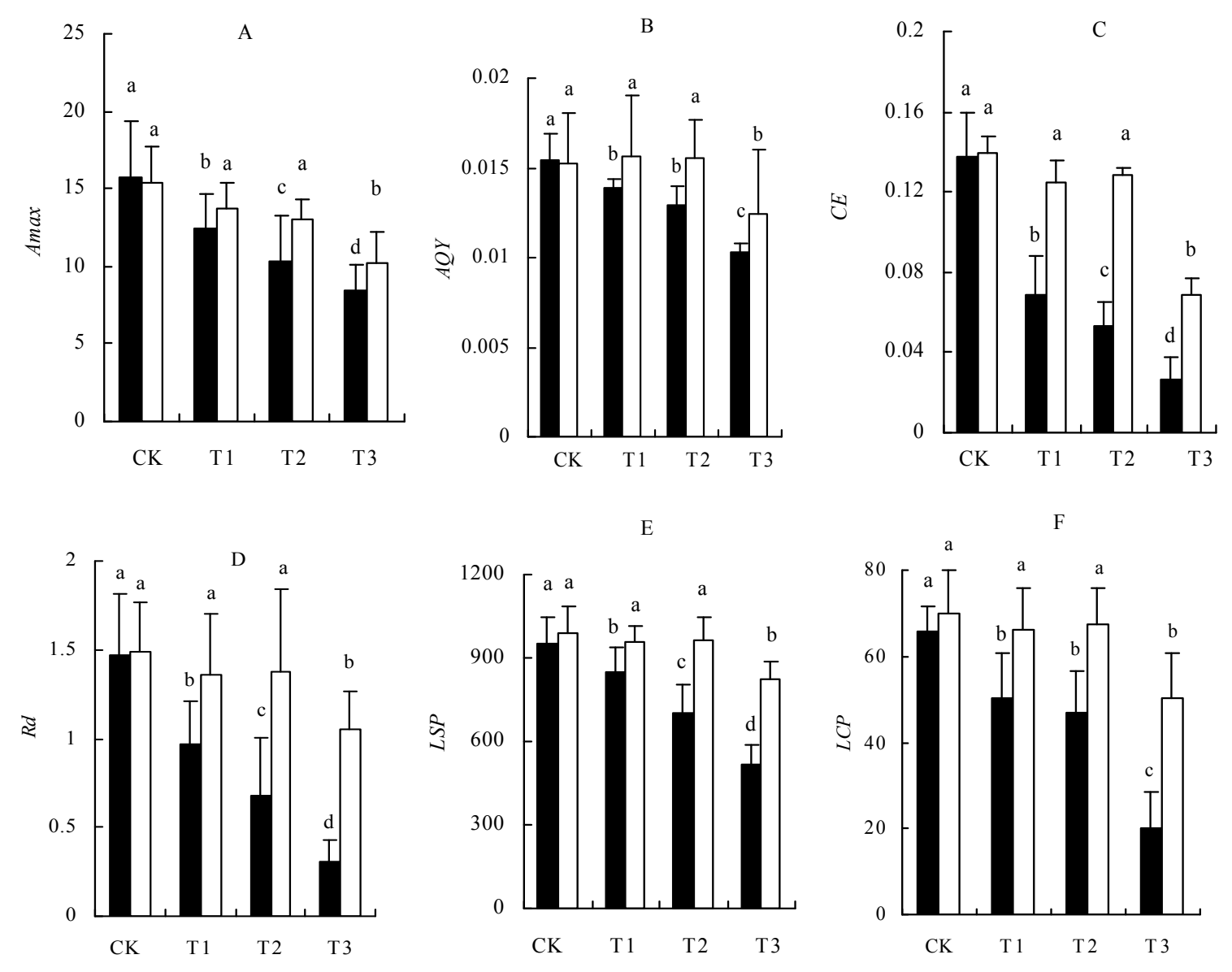

Fig. 3: The comparison of chlorophyll fluorescence parameters between shading stress and light recovery the different letters indicate statistically significant differences at $\mathrm{p}<0.05$ between elevations Shading stress $\square$ Light recovery

The turning point comes on the 4th day when under moderate stress and on the 3rd day under severe stress. Mild stress has always a slowly decline tendency. Others could be able to recover the normal level and there were no significant differences between them $(\mathrm{p}>0.05)$ (Fig. 3A). The non-photochemical energy dissipation of PSII antenna pigment led to the decline of $F_{0}$, while the PSII reaction center which suffers damage or reversible inactivation aroused an increase in $F_{0}$. This research showed that both moderate shading stress and severe shading stress aroused $F_{0}$ decreased firstly and then increase. It told that the decline of energy which was absorbed by PSII antenna pigment flew down into part of photochemistry and the energy increased in the form of heat dissipation and fluorescence (Feijó et al., 2009). With the shading stress time protracted, the photosynthetic structure of strawberry leaf suffers damage to a certain degree. Besides, the turning point of $F_{0}$ comes earlier with the degree of shading stress becoming serious (Fig. 4A).

$F_{m}$ is a fluorescence yield when PSII reaction center is totally closed, reflecting a state of electrical transfer when passed PSII (Razavi et al., 2008). From Fig. 3B, it can be seen that under the same light, with the stress time increased, $F_{m}$ expressed a decline tendency. After 6 days, the decrease of $F_{m}$ varies in different situations, with $35.44 \%$ under mild stress, $39.24 \%$ under moderate stress, $48.11 \%$ under severe stress. Although damage for these stresses could recover after the light was recovered, there were significant differences on damage recover between in severe shading stress group and control group $(p<0.05)$. Others could be able to recover the normal level and there were no significant differences between them $(\mathrm{p}<0.05)$ (Fig. 4B).

$F_{v} / F_{m}$ is the maximal photochemical yield of PSII, reflecting the light energy transformation efficiency of PSII. From Fig. $4 \mathrm{C}$, it can be seen that $\mathrm{Fv} / \mathrm{Fm}$ of strawberry kept 0.81 on average and there was no significant difference among them when given normal light. However, $F_{v} / F_{m}$ gradually declined with shading stress increased in the situation of the same time. Under the same shading stress, $F_{v} / F_{m}$ also gradually declined with shading stress time increased. But $F_{v} / F_{m}$ had 

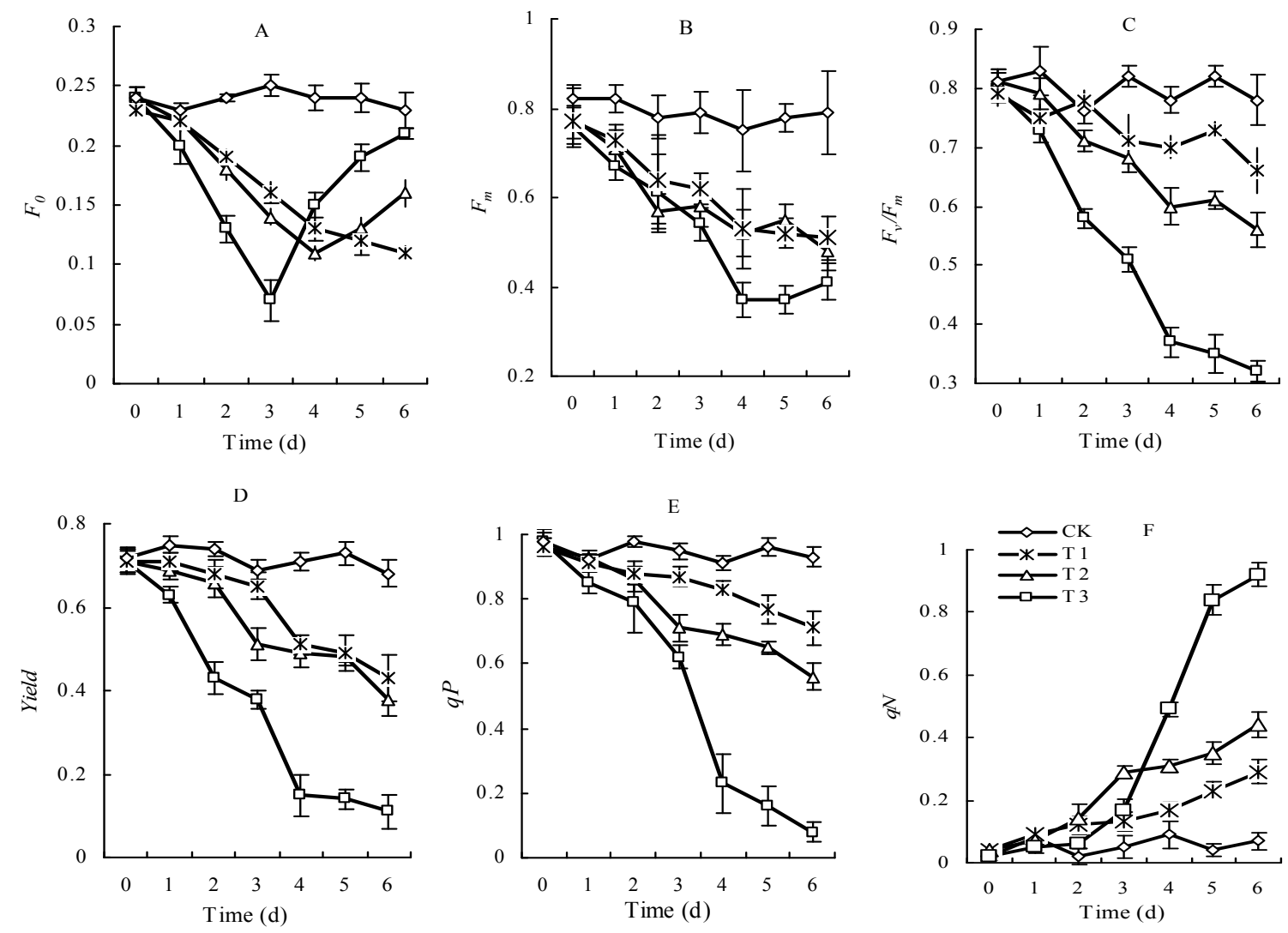

Fig. 4: The change of chlorophyll fluorescence parameters in leaf of $F$. ananassa under shading stress

expressed a significant decrease compared with the control group. The decline of $F_{v} / F_{m}$ varies in different situation, with $15.38 \%$ under mild stress, $28.21 \%$ under moderate stress, $58.97 \%$ under severe stress. Fv/Fm has increased after the light was recovered. However, $F_{\sqrt{ }} / F_{m}$ under severe stress only recovered its $60.12 \%$ of the control group and there were significant differences on damage recover between in severe shading stress group and control group $(\mathrm{p}<0.05)$. Others could be able to recover the normal level and there were no significant differences between them ( $>00.05)$ (Fig. 3C). A number of researches stated that there is a positive correlation between $F_{v} / F_{m}$ and plants growth condition (Razavi et al., 2008)). When plant is in normal condition, the $F_{v} / F_{m}$ has a slight change and varies from 0.75 to 0.85 . $F_{v} / F_{m}$ has a significant decline when plant is in stress condition, which shows that the decrease of photochemical efficiency has seriously limited the normal photosynthesis of strawberry. Combined with the experiment result of $F_{0}$ under shading stress, it showed that shading stress had a partial damage on primary reaction process of strawberry photosynthesis.

Yield is the effective quantum output of PSII, reflecting the actual light energy arresting efficiency of PSII, when part of PSII reaction is closed. It can be directly tested when leaf does not put in adaptation to darkness and as an index of plant photosynthesis electrical transport rate. From Fig. 4D, it can be seen that with stress time increased, yield gradually declined. At the primary shading stage, yield has a slowly decline. After 6 days, it expressed a significant decrease compared with control group $(\mathrm{p}<0.05)$. The decline of yield varies in different situations, with $36.76 \%$ under mild stress, $44.12 \%$ under moderate stress, $83.82 \%$ under severe stress. Although yield has recover after the light was recovered, yield under severe stress only recovered its $32.81 \%$ of the control group. There were significant differences on damage recover between in severe shading stress group and control group $(\mathrm{p}<0.05)$. Others could be able to recover the normal level and there were no significant differences between them $(p>0.05)$ (Fig. 3D).

From Fig. 4E, it can be seen that with the same time, $q P$ gradually declined with stress time increased. At the primary shading stage, $q P$ has a slowly decline. After 6 days, it expressed a significant decrease, compared with control group $(\mathrm{p}<0.05)$. The decline of $q P$ varies in different situations, with $23.66 \%$ under mild stress, $39.78 \%$ under moderate stress, $91.39 \%$ under severe stress. Although $q P$ has recover after the light was recovered, $q P$ under severe stress only recovered its $49.43 \%$ of the control group. There were significant differences on damage recover between in severe shading stress group and control group $(\mathrm{p}<0.05)$. Others could be able to recover the normal level and 
there were no significant differences between them $(\mathrm{p}>0.05)$ (Fig. 3E).

Miyake et al. (2005) suggested that the increase of $q N$ induced by non-radiative dissipation was a photoprotective process. From Fig. $4 \mathrm{~F}$, it can be seen that $q N$ gradually rise with stress time increased. After 6 days, it expressed a significant increscent compared with control group $(\mathrm{p}<0.05)$. Compared with the original value, the increscent of $q N$ varies in different situations, with 7.25 times under mild stress, 11 times under moderate stress, 46 times under severe stress. Although $q N$ has recovered after the light was recovered, $q N$ under severe stress is about 4 times as great as the control group. There were significant differences on damage recover between in severe shading stress group and control group $(\mathrm{p}<0.05)$. Others could be able to recover the normal level and there were no significant differences between them ( $\mathrm{p}>0.05)$ (Fig. 3F).

\section{CONCLUSION}

Because of the shading reduces the light intensity, the net photosynthetic rate per unit area decline greatly, and the greatest net photosynthetic rate and total photosynthetic accumulation in a day decrease. At the same time, shading weakens the $\mathrm{CO}_{2}$ fixation capability, which we can see from the Carboxylation Efficiency (CE) decrease. So when we plan strawberry in the glasshouse, we have to consider not only the dependence light, but also the dependence $\mathrm{CO}_{2}$. Application of $\mathrm{CO}_{2}$ fertilizer will benefit strawberry photosynthetic accumulation and growth. That first shading stress then light recovery can reflect the ability that plant adapts to shading stress and its protection mechanism. After 7 days, the chlorophyll fluorescence parameters $\left(F_{o}, F_{m}, F_{v} / F_{m}\right.$, Yield, $\left.q P, q N\right)$ can recover basically its innate level when strawberry is under mild stress or moderate stress. However, there were significant differences on damage recover between in severe shading stress group and control group $(\mathrm{p}<0.05)$. All these stated that the damage of PSII reaction center can recover its normal level, when the environment is good. But the leaf suffer severe damage, it is hardly to recovery. Thus, it is necessary to pay attention to control light when planted strawberry and often keep soil wet. Besides, for those strawberries which stay in shading condition for a long time, it is vital to supply light to avoid strawberry keeping in serious shading stress.

\section{ACKNOWLEDGMENT}

We would also like to thank the members of the ecology Research Institute of Shaanxi normal university. This study was jointly funded by the National Natural Science Foundation of China (NSFC 31100311), the excellent provincial young fund of Anhui (2012SQRL113ZD) and the doctor fund of Anqing Teachers College.

\section{REFERENCES}

Chazdon, R.L., 1992. Photosynthetic plasticity of two rain-forest shrubs across natural gap transects. Oecologia, 92: 586-595.

Delagrange, S., 2011. Light-and seasonal-induced plasticity in leaf morphology, $\mathrm{N}$ partitioning and photosynthetic capacity of two temperate deciduous species. Environ. Exp. Botany, 70: 1-10.

Feijó, N.S.A., M.S. Mielke, F.P. Gomes, S. Franca and A.O. Lavinsky, 2009. Growth and photosynthetic responses of Gallesia integrifolia (Spreng.) Harms and Schinus terebinthifolius Raddi seedlings in dense shade. Agroforest. Syst., 77: 49-58.

Gianoli, E. and F. Valladares, 2012. Studying phenotypic plasticity: The advantages of a broad approach. Biol. J. Linnean Soc., 105: 1-7.

Jensen, A.M., E.S. Gardiner and K.C. Vaughn, 2012. High-light acclimation in Quercus robur L. seedlings upon over-topping a shaded environment. Environ. Exp. Botany, 78: 25-32.

Miyake, C., S. Horiguchi, A. Makino, Y. Shinzaki, H. Yamamoto and K. Tomizawa, 2005. Effects of light intensity on cyclic electron flow around PSI and its relationship to non-photochemical quenching of chl fluorescence in tobacco leaves. Plant Cell Physiol., 46: 1819-1830.

Oguchi, R., K. Hikosaka, T. Hiura and T. Hirose, 2006. Leaf anatomy and light acclimation in woody seedlings after gap formation in a cool-temperate deciduous forest. Oecologia, 149: 571-582.

Razavi, F., B. Pollet, K. Steppe and M.C. Vanlabeke, 2008. Chlorophyll fluorescence as a tool for evaluation of drought stress in strawberry. Photosynthetica, 46(4): 631-633.

Shimatani, I.K. and Y. Kubota, 2011. The spatiotemporal forest patch dynamics inferred from the fine-scale synchronicity in growth chronology. J. Vegetat. Sci., 22: 334-345.

Valladares, F. and R.W. Pearcy, 1998. The functional ecology of shoot architecture in sun and shade plants of Heteromeles arbutifolia M. Roem, a Californian chaparral shrub. Oecologia, 114: 1-10.

Van Kleunen, M. and M. Fischer, 2005. Constraints on the evolution of adaptive phenotypic plasticity in plants. New Phytol., 166: 49-60.

Wang, L.J., W.B. Jiang and B.J. Huang, 2004. Promotion of 5- aminole-vulinic acid on photosynthesis of melon (Cucumis melo L.) seedlings under lowlight and chilling stress grown conditions. Plant Physiol., 121(2): 258-264.

Wyka, T.P., J. Oleksyn, R. Zytkowiak, P. Karolewski, A.M. Jagodzinski and P.B. Reich, 2012. Responses of leaf structure and photosynthetic properties to intra-canopy light gradients: A common garden test with four broadleaf deciduous angiosperm and seven evergreen conifer tree species. Oecologia, 170: 11-24. 